\title{
Cholestasis Due to Ingestion of Probably Adulterated Health Supplements: A Case Report
}

\author{
Waseem Raja Dar ${ }^{* 1}$, Meenakshi Trikha ${ }^{2}$ and Asim Trikha $^{3}$ \\ ${ }^{1}$ Internist and Physician Specialist, Sanjeev Bansal Cygnus Hospital, India \\ ${ }^{2}$ Department of Biochemistry NC Medical College, Panipat Haryana India \\ ${ }^{3}$ Department of General Surgery Sanjeev Bansal Cygnus Hospital Karnal, Haryana, India
}

*Corresponding author:Waseem Dar, Department of Internal and Critical Care Medicine, Sanjeev Bansal Cygnus Hospital, Railway Road Karnal, Haryana, India, Tel: +918222800676; E-mail: drwaseem.mw@gmail.com

Rec date: May 11, 2016; Acc date: June 06, 2016; Pub date: June 13, 2016

Copyright: (c) 2016 Dar WR, et al. This is an open-access article distributed under the terms of the Creative Commons Attribution License, which permits unrestricted use, distribution, and reproduction in any medium, provided the original author and source are credited.

\begin{abstract}
Health supplements are marketed as non-pharmaceutical means of improving health and are widely used by gym going youth to enhance their musculature. This is largely driven by peer pressure. The constituents of these supplements may not be regulated and may contain anabolic steroids. We here present a case of a young man who developed cholestasis secondary to use of health supplements that possibly contained anabolic steroids.
\end{abstract}

\section{Introduction}

Health supplements are presently used by majority of population to improve their health. These supplements are not prescribed by physicians but manufactured by companies as nonpharmaceutical agents and hence may skip many regulating authorities particularly in developing countries. These supplements may be adulterated with anabolic steroids to enhance their effects.

\section{Case Report}

A 27 year old male presented with yellow discoloration of eyes in the past two days, noticed by his spouse. He also complained of on and off dark color urine and clay colored stools for the last one week. He didn't report any prodromal symptoms, fever, weight loss, abdominal pain or pruritus. He had no recent travel history. For the past six months he increased exercise and was visiting gym three times a week. He denied any drug history but reported regular intake of health supplements for past few months. He denied any intravenous drug use or any transfusions in the past. Past medical and family history was unremarkable. He is a heterosexual and had occasional drinks on week days.

On initial assessment he was conscious and oriented with muscular built and normal vitals. General physical examination showed overweight patient with yellow skin and sclera, no lymphadenopathy, no scratch marks and no stigmata of chronic liver disease. Cardio respiratory, abdominal and neurological examinations were unremarkable.

Laboratory investigations were done. Hemogram was normal. LFT showed conjugated hyperbilirubinemia with cholestatic pattern [Bil 7.2 mg/dl, AST 83 U/L, ALT 73 U/L, ALP 280 U/L, GGT 180 U/L, LDH $275 \mathrm{U} / \mathrm{L}$, and A/G ratio normal). Dipstick for urine was negative for urobilinogen, red blood cells, bilirubin, proteins, glucose and white blood cells. Ultrasound of the abdomen showed normal hepatobiliary system with no evidence of biliary obstruction. The gallbladder was distended but no evidence of calculi. The common bile duct was not dilated. Contrast Enhanced Computer Tomography (CECT) showed normal gallbladder, common bile duct and the biliary system. No evidence of calculi or obstruction was present. Pancreas and pancreatic duct were normal. Liver biopsy showed cholestasis mostly perivenular. Some of the portal tracts showed slight expansion by a minimal increase in chronic inflammatory cells and a slight increase in bile duct profiles. A Periodic-Acid Schiff (PAS) stain showed small numbers PAS-positive Kupffer cells in area showing bilirubin stasis. Connective tissue stain shows no evidence of increased portal fibrosis or of bridging fibrosis. Special stains for iron deposition and for copper associated protein were negative. The appearances were considered to be within the spectrum of appearances seen in bland cholestasis secondary to anabolic steroid use.

Patient was informed about the abnormal liver function tests and the histopathological changes due to anabolic steroid use. Patient didn't report any use of anabolic steroids except for health supplements and hence it was concluded that these supplements were possibly adulterated with anabolic steroids which led to cholestasis in this patient. Patient was advised about complete abstinence from any health supplements in future. Patient was reviewed in clinic after few weeks and blood tests showed normal liver functions.

\section{Discussion}

Anabolic steroids (AS) have been used by the athletes for decades to increase their performance [1]. A current cause for concern is the recent manufacture of analogues of established anabolic steroids to tap into the bodybuilding market. To avoid the statutory controls of countries regarding the manufacture and supply of drugs, these compounds are often widely marketed as nutritional/dietary supplements, examples being DHEA, androstenedione, androstenediol, and their 19-nor equivalents (these steroids are prohormones), and analogues of testosterone and stanozolol called 1-testosterone and prostanozolol, respectively. It is a consequence of their widespread availability in gymanasium and gym instructors advising them for muscle building and fitness. These steroids are supplied for oral administration, and are therefore subject to first-pass metabolism. 
Page 2 of 2

Virtually very less data is available in India on the abuse of anabolic steroids with atheletes in India and also with respect to general population. Hence more studies are to be carried out for any conclusive evidence.

Androgenic anabolic steroids are available in various types and formulations, but, it is the carbon-17-alkylated anabolic steroids, such as methandrostenolone and methyl testosterone- that are more hepatotoxic [2]. The 17-alkylated steroids have been the type of steroid most commonly associated with detrimental effects on the liver [3]. The use of some of these steroids has been withdrawn in the treatment of humans due to adverse effects but AS users still manage to obtain illicit supplies.

Nearly all studies of anabolic steroids users have documented abnormalities in liver function tests with rises in alanine transaminase (ALT) [4] and AST. If the raised levels are attributable to AS then they are usually reversible after stopping steroid use or are transient even if AS use continues [5]. It has been suggested that further studies of AS use in athletes should monitor the enzymes lactate dehydrogenase (LDH) and alkaline phosphate (ALP), which are more specific to the function of the liver [6]. Anabolic steroids are believed to be hepatotoxic, with oral forms of AS exhibiting higher toxicity than equivalent doses of injectable AS [3]. A Research has questioned the level of hepatotoxicity of AS. This study measured increases in the levels of AST and ALT and two additional enzymes, gammaglutamyltranspeptidase (GGT) and creatine kinase (CK). Increases ALT and AST are indicative of tissue damage but are not, in themeselves, representative of damage liver tissue. The study therefore suggests that changes in GGT levels may provide a better indication of liver abnormalities [7]. Cholestasis is a clinical and biochemical syndrome that results when bile flow is restricted. Anabolic steroids are recognized to cause cholestatic jaundice in patients receiving AS to treat a medical illness. In our case, the young patient has also used unspecified health supplements for body building. A Canadian case report has reported the occurrence of cholestasic jaundice, acute pancreatitis and acute injury simultaneously [8].

In conclusion, it is very important to consider about the use of anabolic steroids in young patients with non-specific presentation and abnormal liver functions, so that its further use can be stopped and prevent progressive liver injury. Patients should be followed up in clinic after stopping the use of health supplement with repeat liver function tests.

\section{References}

1. Sjoqvist F, Garle M, Rane A (2008) Use of doping agents, particularly anabolic steroids, in sports and society. Lancet 371: 1872-1882.

2. Dawson RT (2001) Drugs in sport - the role of the physician. J Endocrinol 170: 55-61.

3. Hickson RC, Ball KL, Falduto MT (1989) Adverse effects of anabolic steroids. Med. Toxicol. Adverse Drug Exp 4: 254-271.

4. Freed DLJ, Banks AJ, Longson D, Burley PM (1975) Anabolic steroids in athletics: Crossover double-blind trial on weightlifters. Br Med J 2: 471-473.

5. Lamb D R (1984) Anabolic steroids in athletics: How well do they work and how dangerous are they? Am J Sports Med 12: 31-38.

6. Kibble WM, Ross MB (1987) Adverse affects of anabolic steroids in athletes. Clinical Pharmacology 6: 686-692.

7. Dickerman RD, Pertusi RM, Zachariah NY, Dufour DR, Mc Conathy WJ (1999) Anabolic steroid-induced hepatotoxicity: Is it overstated? Clinical Journal of Sport Medicine 9: 34-39.

8. Rosenfeld GA, Chang A, Poulin M, Kwan P, Yoshida E (2011) Cholestatic jaundice, acute kidney injury and acute pancreatitis secondary to the recreational use of methandrostenolone: a case report. J Med Case Rep 5: 138 